\title{
Therapeutic drug monitoring in oncology: does it have a future?
}

"...clinical application of cancer drugs represents a unique toxicological and clinical compromise where the seriousness of the disease, similarity of target and normal body cells, and overt toxicity of the drugs applied are uncomfortable bed fellows."
Cancer is a major health issue worldwide and chemotherapeutic drugs are a major component of the treatment of this disease, particularly in its advanced stages. In any disease, the net efficacy of an administered pharmaceutical is the sum of its therapeutic and toxic actions. For many drugs and in the majority of patients, the concentrations at which serious toxicity is seen is many times greater than the concentration at which efficacy is evident. However, clinical application of cancer drugs represents a unique toxicological and clinical compromise, where the seriousness of the disease, similarity of target and normal body cells, and overt toxicity of the drugs applied are uncomfortable bed fellows. Because the majority of anticancer drugs are cytotoxic in nature and have little specificity, the optimal dose for a given patient will likely exist in a very narrow range. Hence, it might be anticipated that therapeutic drug monitoring (TDM) represents a significant opportunity to improve the standard of care for cancer treatment. However, with few exceptions, TDM has not found its way into routine clinical oncology practice. Why is this and where might the future lie for TDM in cancer treatment?

\section{Therapeutic drug monitoring}

The fundamental tenet of TDM is that alterations in a drug-administration scheme can be related to some analytical measure of a drug that then predictably correlates with an important metric of action (e.g., tumor kill or hematological toxicity). TDM is also generally accepted to be valuable if the drug has a narrow therapeutic range, the therapeutic effect is difficult to define and there is wide variation in pharmacokinetics between patients [1]. While at first glance this perspective appears straightforward, there are a number of very fundamental practical and scientific challenges inherent in this concept, including practical/ clinical factors, therapeutic/pharmacokinetic considerations and analytical issues.

\section{Practical/clinical factors}

Despite some recent advances, the clinical use of any drug treatment is still largely targeted at a general patient population. In particular, standard doses are employed that make a number of assumptions about a patient. Recognizing that variations in patient size, fat and blood volume could significantly (and even fatally) impact on cancer treatments, it became common to attempt to normalize the administration of intravenous cancer drugs by dosing on the basis of body surface area or occasionally by absolute weight [2]. While the scientific rationale underlying this approach is surprisingly weak and with different formulae available for body surface area calculation, by and large, such methods, combined with the expertise and experience of the oncology teams, appear to serve patients with average dimensions reasonably well. But can we do better?

"...both aspects of treatment individualization (biochemical and pharmacological) will have to be managed if the most efficacious treatment is to be provided..."

Adding to the complexity, the average cancer patient, who is typically elderly, will be treated with a combination of cancer drugs, with additional agents also being employed to manage side effects (e.g., steroids) and comorbidities (e.g., circulatory disease or infection). In other words, the average patient may have five to ten pharmaceuticals in their system at any one time, with potential for drug interactions and providing significant analytical complexity.

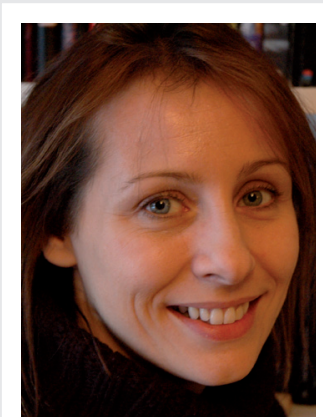

Gillian McMahon

Author for correspondence National Centre for Sensor Research, School of Chemical Sciences, Dublin City University, Glasnevin, Ireland Tel.: +353 17005914

Fax: +35317005503

E-mail: gillian.mcmahon@ dcu.ie

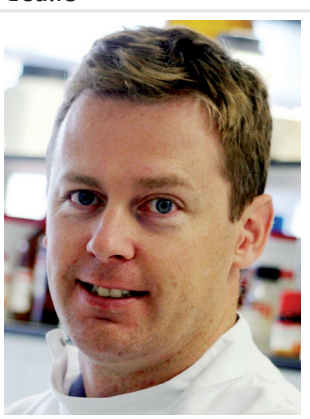

\section{Robert O'Connor}

National Institute for Cellular Biotechnology, Dublin City University, Glasnevin, Ireland E-mail: robert.oconnor@ dcu.ie

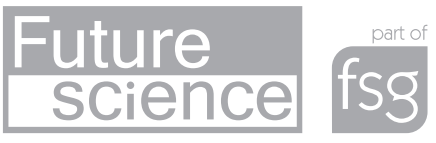


In addition, cancer treatment is evolving to become more targeted and individualized, with agents such as trastuzumab and lapatinib targeting specific molecular abnormalities identified by increasingly complex biochemical pathology [3]. However, the focus of the major pharmaceutical companies to date has been almost entirely on biochemical instead of pharmacological individualization; treating on the basis of the molecular/biochemical characterization of the tumor, rather than individualized dose adjustment, which would take account of the apparent differences in absorption, distribution, metabolism and elimination characterized by the targeted agent in the patient. Further research will likely demonstrate that both aspects of treatment individualization (biochemical and pharmacological) will have to be managed if the most efficacious treatment is to be provided to a specific patient.

\section{Therapeutic/pharmacokinetic considerations}

The long-held rationale for using harmful agents is that cancer represents a proliferative disease and anything that kills growing cells might be expected to halt tumor growth. Clearly, cytotoxics will have significant side effects because there are many actively growing normal cells and tissues even in a diseased body. Based on their toxicological actions alone, it is clear that the majority of anticancer agents are strong candidates for TDM use. However, and perhaps surprisingly, there is a dearth of data for many established anticancer agents on their concentration-effect relationship for toxicity and, especially, for efficacy [4]. The tumor environment is complex and extremely variable, and most drugs in use may have multiple extratumoral and intracellular targets, which can range from the tumor vasculature down to key specific enzymes controlling the life and death of the cell. With such complexity, it is difficult to identify what markers we might employ as surrogates of effect (pharmacodynamic markers). Identification of such correlates is clearly important in the ability to quantify effect [4].

Allied to this, the methods of assessment of cancer drug action are also complex. These can range from general factors, such as overall survival, to generalized markers of toxicity that ignore whether the drug is working on the tumor or not. While survival is clearly the most important metric, for most drugs, TDM seems to have most predictive power when correlated with specific measures of toxicity. In other words, the TDM approach seems to be the most useful for reducing incidence and severity of toxicity instead of augmenting efficacy rates. Curiously, evidence, both anecdotal and quantitative, suggests that toxicity itself may be a useful predictive correlate for antitumor action and, in at least some cases, the patients with the most acute toxicity can also be those who demonstrate the most durable responses [5]. Both the therapeutic and toxicological response to cancer treatment should, in theory at least, generate specific patterns of response markers in the bloodstream, also known as biomarkers. If such biomarker profile responses are durable (qualitatively or quantitatively), then melding of TDM with biomarker research may permit generation of response algorithms that empirically eliminate the need for drug determination. However, research in this area is still in its infancy because the first trials validating the therapeutic utility of predictive biomarkers as a modifier in the choice of which therapy to use have only recently commenced and we may be decades away from using biomarkers as a dose-optimization tool in the clinical setting [6]. Advancement of this area will require specific research that identifies useful TDM and toxicity and response biomarkers and their correlations with dose. In other words, a panel of markers that indicate, in an appropriately timely fashion, whether the drug treatment schedule is generating manageable toxicity and response and giving information that can be used to tailor dose up or down arising from the measurements of these markers.

\section{"...evidence, both anecdotal and quantitative, suggests that toxicity itself may be a useful predictive correlate for antitumor action..."}

An obvious corollary to the basic concept of cancer drug TDM is that the amount of drug that a cancer cell is exposed to should, in some way, correlate with activity. However, the practicalities of advanced cancer treatment greatly limit investigating or applying this concept. Cancer is often a metastatic disease, spread in unknown areas in the body, where the exact location and number of tumor cells is impossible to ascertain. Indeed, since the most effective cancer drugs induce cell death (typically apoptosis) in target cells, we do not even know the most appropriate time to measure intracellular/ tumor drug concentrations, as death will clearly impact on drug measurement. 
Our most convenient source of drug-level data is analytical determination of levels in the blood (typically serum or plasma quantification). However, it must be remembered that the drug concentration in plasma or serum will often be an indirect measure of the amount of drug affecting the target site or acting at the cellular level, since the blood supply may be remote from the cancer. This is particularly confounding in the case of serum cytotoxic quantitation because the intravenous administration of such agents means that the drug levels in the blood decrease very rapidly as the agent is taken up by numerous tissues. The situation with these drugs is even more complex since most are highly plasma protein bound and some, but not all, authors feel that the effective drug fraction is the free component [7]. So should we measure free drug, bound drug and/ or total drug as a baseline for determinations? And how often should we/can we make such determinations since sampling involves ongoing patient discomfort?

\section{“..it must be remembered that the drug concentration in plasma or serum will often be an indirect measure of the amount of drug affecting the target site or acting at the cellular level since the blood supply may be remote from the cancer."}

Therapeutic drug monitoring first demonstrated therapeutic promise decades ago when researchers began to mathematically summarize the analytical data resulting from blood druglevel determinations using standard pharmacokinetic approaches. Because of the nonlinear relationship and temporal delay between drug exposure and effect, to date only largely observational approaches appear to identify which constants (e.g., AUC) correlate with useful measures of drug action, and there can be no doubt that better identification of pharmacokinetic surrogates for toxicity/efficacy can be useful. For example, acute toxicity of carboplatin can be predicted by determination of the AUC for this agent. However, platinum quantitation is not available in the majority of clinical settings and, thus, a further approximation exploits the Calvert formula, which makes use of the patient's pre-existing excretory function (glomerular fltration rate) to predict the AUC and individualize dose, an approach that significantly reduces the incidence of toxicity.
Notwithstanding our knowledge of cancer pharmacokinetics, we are only now beginning to more routinely apply sufficiently complex mathematical strategies to make use of the analytical data that TDM could provide. Application of such methods, such as Bayesian analyses, has the capacity to increase the accuracy of our determinations and greatly reduce the numbers of samples taken from the patient. Combining such approaches with emerging drug-modeling technology has huge potential both in the development and routine clinical application of cancer drugs [8]. However, such methods will need to migrate from their current focus on the variables affecting a single drug, since cancer is now rarely treated by monotherapy. It is clear that the pharmaceutical companies developing and testing existing and new anticancer pharmaceuticals have the capability to derive much more useful information from early clinical (and even preclinical) analytical and pharmacokinetic determinations. Also, regulatory agencies such as the US FDA are increasingly requiring the assimilation of more complex datasets in order to provide more predictive information. However, even now, such approaches are largely confined to predictive estimation of the impact of single drugs, while the majority of patients will receive concurrent treatment with two or more anticancer agents (ignoring the concomitantly administered agents most likely being used to manage other morbidities present).

\section{Analytical issues}

As already mentioned, the average cancer patient will be administered a combination of anticancer agents, along with additional drugs for managing side effects and other disorders. In the case of some chemotherapy drugs, such as the anthracyclines or taxanes, the active species is the parent drug; however, for other agents, especially alkylating agents and purine/pyrimidine antagonists, the parent drug must be converted to a shortlived species, which will be covalently incorporated in the cancer cell. In this case, determination of the parent drug or specific metabolites may only be a surrogate for the actual amount of active species present.

The actual determination of cancer drugs in itself can also be challenging. In general, agents are administered intravenously and have very high tissue uptake and body elimination. Hence, concentrations can range from micromolar to nanomolar over only a few hours, so any analytical method must be simultaneously sensitive and 
have a broad linear range. Most anticancer agents are highly plasma protein bound and, particularly in the case of cell or tissue drug determinations, the drug may be highly bound to organic tissue. Extraction methods must, therefore, be very efficient if accurate estimations of drug level are to be achieved. Many cancer drugs are also unstable under certain conditions of light, $\mathrm{pH}$ or solubility, and, therefore, the analyst may need to rapidly analyze samples and/or provide additional buffers to preserve the integrity of the analyte.

The determination of chemical (small molecule) cancer agents has been greatly facilitated by advances in analytical instrumentation. In particular, liquid chromatography-mass spectrometry (LC-MS) has had a greater impact in cancer drug determination than other classes of pharmaceuticals [9]. The ideal analytical methodology for cancer drugs should be reasonably rapid, allow for high sample throughput, be robust and sensitive and allow for quantitation of multiple drugs and metabolites simultaneously.

\section{"Molecular individualization of cancer therapy only works if there is also pharmacological individualization, with its inherent dependence on therapeutic drug monitoring."}

While the analytical 'solution' for TDM applications of many cancer drugs may reside in MS techniques, such technology comes at a high cost in terms of equipment and the expertise to use and apply the resultant data. All public health services are constrained by resources and doubtless a clinician eager to employ TDM might reasonably seek to balance its potential impact and cost against the proven success of more aggressive toxicity-management regimens. The advent of cheaper, more user-friendly equipment combined with an increasing acceptance within hospital biochemistry departments of LC-MS to classify common metabolic disorders may be starting to bring such facilities within easier reach of clinicians willing to embrace TDM.

Antibody-based therapeutics, such as trastuzumab and bevacizumab, have now established a role in the treatment of advanced malignancies. With their distinctly different pharmacology, these agents also present specific analytical challenges. Current quantitation methods are largely based on ELISA-based technologies to measure circulating levels of the agent. While ELISAbased quantitation can have inherent technical limitations, pharmacokinetic analyses conducted as part of the drug-development process have shown that these agents have extremely long half-lives, largely negating the need for TDMbased dose adjustment. The practical limitations of ELISA also mean that tumor levels of these therapeutic antibodies cannot be determined. Improved and more economical methods of antibody determination, such as those based on emerging MS methods, may permit better modeling of therapeutic antibody distribution within the tumor and associated stroma, but a clear role for TDM in routine clinical application of therapeutic antibodies is not yet evident.

\section{Future perspective}

As emphasized by other authors in the field, it is important to stress that implementation of TDM in the oncology setting, while offering huge benefits to patients, is complex and needs buy-in from the many different disciplines involved in treating cancer patients [4]. A quality-driven process is vital and every step from drug administration, to collection and handling of blood samples, to meticulous and sensitive determination of the analytes of interest, to interpretation of the data, must be carefully validated if TDM is to deliver to its full potential.

While individual, high-quality research reports have clearly demonstrated specific uses of TDM methodology, perhaps the factors necessary to properly capitalize more generally on such approaches are only now aligning appropriately.

The future promise of TDM in the oncology setting most probably lies in a less ambitious, more pragmatic approach, identifying cohorts of patients where TDM can be demonstrated to broadly improve treatment efficacy in a cost- and time-effective manner. Objective evidence still points to the major role for TDM being in the early identification and management of adverse drug reactions. Taking account of all factors, especially economics, it appears unlikely that all cancer patients could or should have TDM methods applied to their treatment. However, there are clear arguments for applying this approach to specific subsets of patients, such as those showing unexpected life-threatening toxicities, those with pre-existing morbidities that compromise use of normal treatment protocols, the elderly, those significantly outside normal body dimensions, particularly the obese (an increasing cohort in oncology clinics) and the very young (a group poorly researched in conventional drug-evaluation process). 
The necessary analytical equipment and expertise is becoming more readily available to clinicians on their doorsteps, which should facilitate consideration of TDM as an available tool. Research is also increasing the power, sensitivity and speed of the drug-quantification process, making it more applicable in the conventional clinical application, as opposed to specialist centers. And development of more accessible pharmacological modeling tools will complement the analytical advances currently emerging, allowing better identification of key determinants of response and, in particular, toxicity.

New approaches to multimodality treatment may also play a part. Neoadjuvant drug administration (giving cancer drugs before surgery) is demonstrating improved response in specific malignancies. Quantification of drug levels in the recovered tumor material may permit better determination of the actual relationship between drug dose and tumor delivery.

Further into the future, TDM may rely less on actual drug quantitation and/or be augmented by analysis of pharmacodymanic markers (including nucleic acid and protein biomarkers), which give quantitative information regarding both the toxicity and efficacy response to the treatment, but a significant research effort will be required to identify and validate the utility of such biomarkers.

Fundamentally, we must ask ourselves: TDM in oncology - why bother? The answers are perhaps more pragmatic that we might suspect:

- Many, if not most of us will get cancer, particularly as the population ages;

- The drugs used to treat the common forms of this disease still have huge potential for causing serious injury and we need to use advanced, intelligent administration (informed by TDM methodology) to optimize the therapeutic potential and reduce side effects and toxicity;

- Pharmacoeconomics is playing an increasing role in the treatment equation and we must take all reasonable steps to reduce costs while simultaneously giving the best treatment available;

- Technological advances that make cancer treatment easier/more effective (especially with new molecularly targeted therapeutics) will also mean that we need to monitor compliance more routinely (for the patient, but also for the drug-development process itself) Advances in analytical technology, detector sensitivity and mathematical modeling are all making more sophisticated TDM resources readily available to the treating clinician.

Ultimately, individualization is the key for the future drug treatment of cancers. The molecular pathologist will tell us which drug is most appropriate and the analytical pharmacologist or bioanalyst will say what the correct dose is. Molecular individualization of cancer therapy only works if there is also pharmacological individualization, with its inherent dependence on TDM.

\section{Financial \& competing interests disclosure}

The authors have no relevant affliations or financial involvement with any organization or entity with a financial interest in or financial conflict with the subject matter or materials discussed in the manuscript. This includes employment, consultancies, honoraria, stock ownership or options, expert testimony, grants or patents received or pending, or royalties.

No writing assistance was utilized in the production of this manuscript.

\section{Bibliography}

1 Suthakaran C, Adithan C. Therapeutic drug monitoring - concepts, methodology, clinical applications and limitations. Health Admin. XIX(1), 22-26 (2006).

2 Mathijssen RH, de Jong FA, Loos WJ, van der Bol JM, Verweij J, Sparreboom A. Flat-fixed dosing versus body surface area-based dosing of anticancer drugs in adults: does it make a difference? Oncologist 12(8), 913-923 (2007).

3 Petrelli A, Giordano S. From single- to multi-target drugs in cancer therapy: when aspecificity becomes an advantage. Curr. Med. Chem. 15(5), 422-432 (2008).
4 Hon YY, Evans WE. Making TDM work to optimize cancer chemotherapy: a multidisciplinary team approach. Clin. Chem. 44, 388-400 (1998).

5 Chintamani C, Singhal V, Singh JP, Lyall A, Saxena S, Bansal A. Is drug induced cytotoxicity a good predictor of response to new adjuvant chemotherapy in breast cancer? A prospective clinical study. BMC Cancer 4 , 48 (2004).

6 Cardoso F, Van't Veer L, Rutgers E, Loi S, Mook S, Piccart-Gebhart MJ. Clinical application of the 70-gene profile: the MINDACT trial. J. Clin. Oncol. 26(5), 729-735 (2008).
7 Sparreboom A, Nooter K, Loos WJ, Verweij J. The (ir) relevance of plasma protein binding of anticancer drugs. Neth. J. Med. 59(4), 196-207 (2001).

8 Alnaim L. Therapeutic drug monitoring of cancer chemotherapy. J. Oncol. Pharm. Pract. 13(4), 207-221 (2007).

9 Rochat B, Fayet A, Widmer N et al. Imatinib metabolite profiling in parallel to imatinib quantification in plasma of treated patients using liquid chromatography-mass spectrometry. J. Mass Spectrom. 43(6), 736-752 (2008). 